\title{
Análise de Desempenho na Virtualização de Servidores Para Uso em Banco de Dados PostgreSQL
}

\author{
Elmo B. de Faria ${ }^{1}$, Murilo N. H. Kawanami1 \\ ${ }^{1}$ Instituto de Computação - Universidade Federal de Mato Grosso (UFMT) \\ elmodic.ufmt.br, murilo.kawanamidaluno.ic.ufmt.br
}

\begin{abstract}
Server virtualization is a technique widely used in large companies in order to have several simultaneous environments using the same structure. Over the years, this technology has been improved, both by hardware manufacturers and developers of these technologies, reaching a result as is currently seen in KVM, XenServer and Vmware. In order to obtain the knowledge of which would be the most efficient virtualizer, performance tests will be applied in each of the three virtualizers, which will extract the maximum performance from each one.
\end{abstract}

Resumo. A virtualização de servidores é uma técnica muito utilizada em grandes empresas com a finalidade de possuir diversos ambientes simultâneos utilizando uma mesma estrutura. Ao longo dos anos, esta tecnologia foi sendo aprimorada, tanto por parte de fabricantes de hardware, como por parte dos desenvolvedores destas tecnologias, chegando a um resultado como é visto atualmente no KVM, XenServer e Vmware. Com a finalidade de obter o conhecimento de qual seria o virtualizador com maior eficiência, testes de performance serão aplicados em cada um dos três virtualizadores, o qual irá extrair a máxima performance de cada um.

\section{Caracterização do Problema}

A virtualização não deve ser utilizada em qualquer situação, indiscriminadamente ou se apresenta como uma solução para as demandas de TI. Uma questão de difícil resposta, mas põe a discussão nos inúmeros centros de processamento de dados. Cada organização possui características e necessidades próprias que devem ser cuidadosamente analisadas e consideradas antes de se adotar a virtualização. Dois aspectos são fundamentais: o projeto de virtualização e a virtualização e a infraestrutura de hardware. Um projeto de virtualização deve considerar o levantamento da infraestrutura atual e os objetivos que se pretende atingir. Fazem parte dessa etapa tanto análises de recursos financeiros e de seu retorno, como análises técnicas. Segundo, apesar de cada organização possuir características e infraestruturas próprias, as plataformas existentes podem ser genericamente classificadas em três grandes grupos: servidores, dispositivos de armazenamento (storages) e infraestrutura de rede. O objetivo então é detalhar esses dois aspectos, iniciando pelas questões relacionadas com o projeto de virtualização. Um projeto de virtualização merece um cuidado especial. Definir corretamente a infraestrutura para suportar as aplicações no ambiente virtualizado é uma tarefa complexa, pois envolve diversas variáveis. Também se deve levar em consideração a necessidade de crescimento do ambiente e o consumo de recursos quando do uso de

\footnotetext{
* Projeto realizado na Escola Superior de Redes (ESR - unidade Cuiabá), vinculado ao Instituto de Computação da Universidade Federal de Mato Grosso
} 
algumas funcionalidades. Se a infraestrutura já existe, compartilhá-la com a virtualização requer um estudo sobre as suas reais possibilidades [Veras, M. E Carissimi 2015].

As principais etapas a serem consideradas em um projeto de virtualização estão resumidos em:

1. Educação;

2. Planejamento da capacidade (Capacity Planning);

3. Escolha de fornecedores;

4. Projeto;

5. Implementação (Deployment);

6. Gerenciamento;

O projeto de virtualização se inicia com a educação e conscientização sobre os desafios e necessidades de sua organização. Em seguida, a etapa de planejamento da capacidade é necessária para atender as demandas da organização. Com base nas informações coletadas e nos cenários é feita a opção pelo fornecedor do software de virtualização.

Uma solução de virtualização de servidores pode incluir tecnologias avançadas como tolerância a falhas, balanceamento de carga e redução do consumo de energia. Porém, todas estas tecnologias têm um custo associado em termos de complexidade e recursos necessários. Estes recursos aprimoram a operação da infraestrutura, reduzindo o tempo de interrupção e consequentemente melhorando o tempo de resposta. No entanto, podem não ser determinantes a todos os projetos.

\section{Objetivos}

Novas áreas de conhecimento exigem uma infraestrutura de TI ágil e flexível para suportar a dinâmica dos processos de negócio. O uso da virtualização pode trazer grandes benefícios para a organização com a adequação da sua infraestrutura de TI ao negócio, mas requer planejamento e aquisição de novos recursos. E tem como objetivo principal diminuir a infraestrutura de servidores, com uso racional dos recursos de Hardware e Software, minimizando custos.

Objetivos específicos da virtualização:

- Reduzir o Total Cost of Ownership (TCO) ou custo total de propriedade

- Reduzir o uso do espaço físico

- Reduzir o consumo de energia

- Isolamento de ambientes de testes, desenvolvimento e produção

- Flexibilizar a disponibilização de novos servidores

- Padronizar as plataformas

- Definir Gerenciamento centralizado

- Simplificar o uso de High Availability (HA) e recuperação de desastres

- Criar um ambiente de computação em nuvem e data center dinâmico

\section{Metodologia}

Foram utilizados os computadores para implementar máquinas virtuais utilizando o software VMware, XEN Server, KVM e seus aplicativos para controle e gestão de cursos 
presenciais e a distância. Este projeto avalia três plataformas distintas de software, são elas:

1. O VMware vSphere, o primeiro sistema operacional para computação em nuvem (cloud computing). Ele utiliza os recursos da virtualização para transformar datacenters em infraestruturas de computação em nuvem, consideravelmente simplificadas, e permite que as organizações de TI forneçam a próxima geração de serviços flexíveis e confiáveis, através de recursos internos e externos com segurança e baixo risco. Baseado nos recursos da plataforma VMware Infrastructure, o vSphere permite reduzir os custos operacionais e de capital, além de aumentar o controle sobre o fornecimento de serviços de TI, enquanto preserva a flexibilidade de escolha entre qualquer tipo de sistema operacional, aplicativo e hardware hospedados internamente ou que utilizam recursos externos.

2. O XEN XenServer é uma plataforma de virtualização de servidor abrangente, com recursos de classe empresarial, criada para o fácil manuseio de vários tipos de carga de trabalho, sistemas operacionais mistos e armazenamento ou configurações de rede. Para o aplicativo mais exigente e os casos de uso de virtualização de desktop, a sua capacidade de expansão líder do setor e o desempenho sob carga podem atender às implantações de maior porte de XenApp ou XenDesktop. Para o setor de TI trás o benefício de recursos exclusivos, como gráficos virtualizados aprimorados com NVIDIA e Intel, e segurança avançada de carga de trabalho com APIs de inspeção direta e com proposta de reduzir os custos e a complexidade da infraestrutura virtual.

3. O KVM é uma tecnologia de virtualização open source baseado em Linux, que possui forte apoio da empresa RedHat, através dele é possível transformar o servidor linux em um hipervisor de tipo-1 (bare-metal), e o KVM dispõe de todos os componentes necessários para a virtualização. Segundo a RedHat, o KVM faz parte do Linux, assim como o contrário também é válido, assim herdando diversas características do Linux [Jung, I.H. e Lee, S.H. e Eom, Y.I 2012].

\subsection{Local do experimento}

Esse projeto será realizado na Escola Superior de Redes (ESR) anexo ao Instituto de Computação da Universidade Federal de Mato Grosso (UFMT).

\subsection{Equipamentos utilizados}

\subsubsection{Servidores}

Neste projeto, além da infraestrutura de rede da ESR (Escola superior de redes) que conta com dois laboratórios de 20 máquinas e sistema de videoconferência, serão utilizados 3 computadores Dell possuindo um processador Intel(R) i5-2400 (6MB Cache, 3.4GHz), $16 \mathrm{~GB}$ de memória RAM e capacidade de armazenamento de $250 \mathrm{~GB}$.

\subsubsection{Ativos de redes}

Serão utilizados ativos de redes disponíveis na ESR, como switch, link com a internet. Este sistema foi utilizado para instalação do sistema operacional, das ferramentas de software e atualização das ferramentas, remotamente via protocolo SSH. Os testes dos virtualizadores e do banco de dados foram realizados de forma isolada, para não interferir nos dados obtidos. 


\subsection{O Cenário de testes}

O cenário proposto da infraestrutura de rede é apresentado na Figura 02, ela é composta pelos servidores relatados no item 3.2.1., possuindo configurações idênticas, distinguindo-se através de seus respectivos sistemas operacionais, serviços instalados e virtualizadores estudados. Para cada máquina virtual instanciada, será necessário alocar uma quantidade de memória RAM, disco e a interface virtual, que será compartilhada ou alocada, a fim de atender a demanda do sistema operacional que ali será instalado mais os serviços que ele proverá.

O procedimento será avaliado em ambos os servidores, ao longo da utilização dos mesmos para realização dos testes, vão ser feitas medições para avaliar o desempenho do banco de dados PostgreSQL.

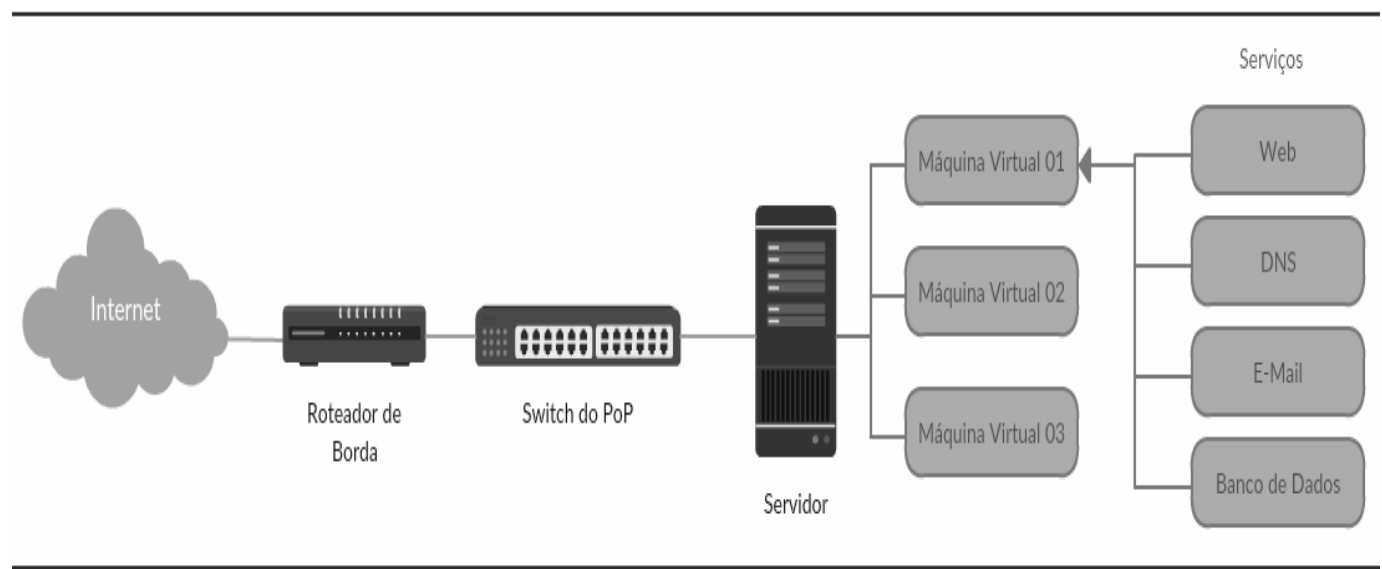

Figura 2 - Cenário proposto dos serviços provido por meio do servidor virtualizado para Xen-server e VmWare (Fonte: [Veras, M. E Carissimi 2015])

Para que os testes possuam o mesmo ambiente homogêneo de testes, em cada máquina virtual instanciada, será alocada 60GB de armazenamento, $8 \mathrm{~GB}$ de memória RAM, e 1 vCPU. Em torno destas configurações, será utilizado o Ubuntu Server 16.04.6 LTS como sistema operacional para os testes [Lee, B.Y. e Park, J.H., Yoo 2014].

\subsection{Teste do sistema}

Para testar, validar e homologar a proposta desse projeto, utilizou-se alguns ensaios no que tange a verificação da performance e consumo de recursos computacionais, para isso, sendo dividido inicialmente em duas fases, apresentadas em detalhes nas próximas sessões. [Seol, K., Hwang1 e H., Moon1, I. e Kwon1 O. e Kim, B. 2014].

A primeira fase tem como foco quantificar o consumo de recursos, e para isso será instanciado uma máquina virtual/container com as mesmas configurações nas três tecnologias virtualizadoras - VMWare, Xen-server e KVM. Os critérios a serem analisados nesta fase tem por objetivo quantificar o uso de memória RAM e processamento necessários para seja possível fornecer o mesmo tipo de serviços nos dois cenários. A fim de realizar os testes, serão utilizados o pgbench - um software integrado ao banco de dados PostgreSQL - e o SAR, ferramenta de monitoramento do sistema presente no Linux. Através destas duas ferramentas, será possível extrair dados de consumo e pontuação dos testes. Os dados gerados ficam armazenados em arquivos que geram os gráficos apresentados na sessão seguinte. 
A segunda fase, tem como foco verificar o desempenho e a consistência do sistema virtualizado. Para isso, serão instalados e configurados os serviços de DNS e WEB para mensurar sua estabilidade e performance no tempo.

\section{Resultados práticos}

Utilizando-se do pgbeng, foi executada uma simulação que tem por objetivo utilizar-se de uma base de dados e realizar um teste de estresse no banco de dados, simulando um tráfego intenso e uma alta quantidade de acessos. A ferramenta foi configurada de modo a realizar a simulação de 100 acessos simultâneos ao banco de dados, cada qual executando operações, por um período pré-definido de 300 segundos.

Durantes os testes, foi possível observar uma ampla diferença entre os virtualizadores testados, tornando esta diferença mais evidente com os gráficos gerados pelo SAR, e pelos resultados obtidos através do pgbench. Nas figuras 3, 5 e 7 são exibidos os gráficos produzidos pelo SAR, expressando além do consumo total de recursos da CPU, o consumo individual de cada parte do sistema. No primeiro gráfico de cada figura, é exibida o consumo de recurso, e dentre os maiores consumidores de recursos, estão o recurso do usuário, expresso na cor verde, o consumo pelo próprio sistema, expresso na cor vermelha, consumo de software, expresso pela cor azul e tempo de espera do sistema para executar a próxima tarefa, expressa pela cor rosa. No segundo gráfico de cada figura, o gráfico representa a porcentagem de recursos livre da CPU, fazendo uma contraposição em relação ao primeiro gráfico.

\subsection{KVM}

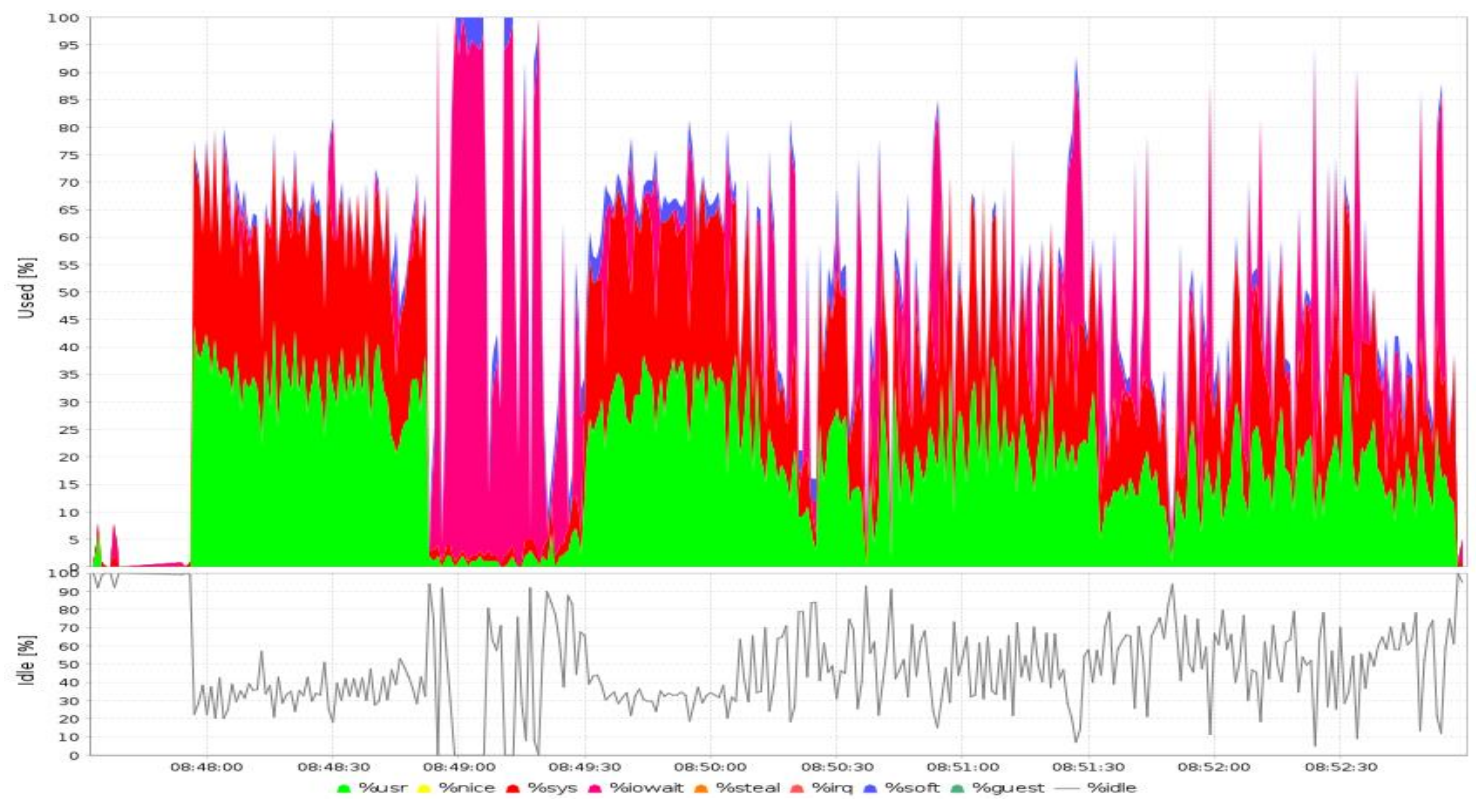

Figura 3 - Gráfico de uso da CPU pelo KVM (Fonte:Autor)

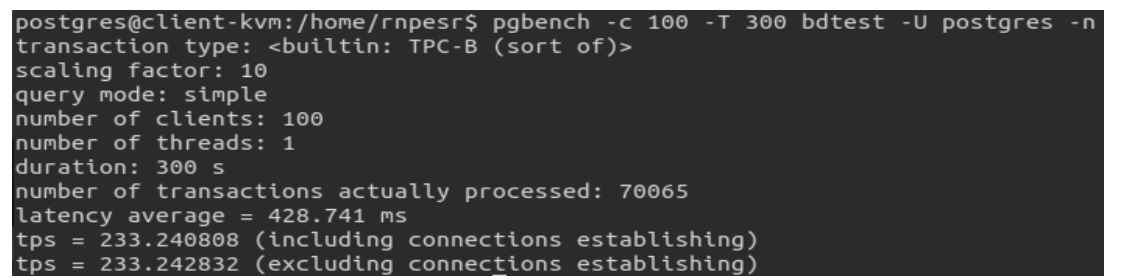

Figura 4 - Resultados de benchmark do pgbench no KVM (Fonte:Autor) 


\subsection{Vmware}

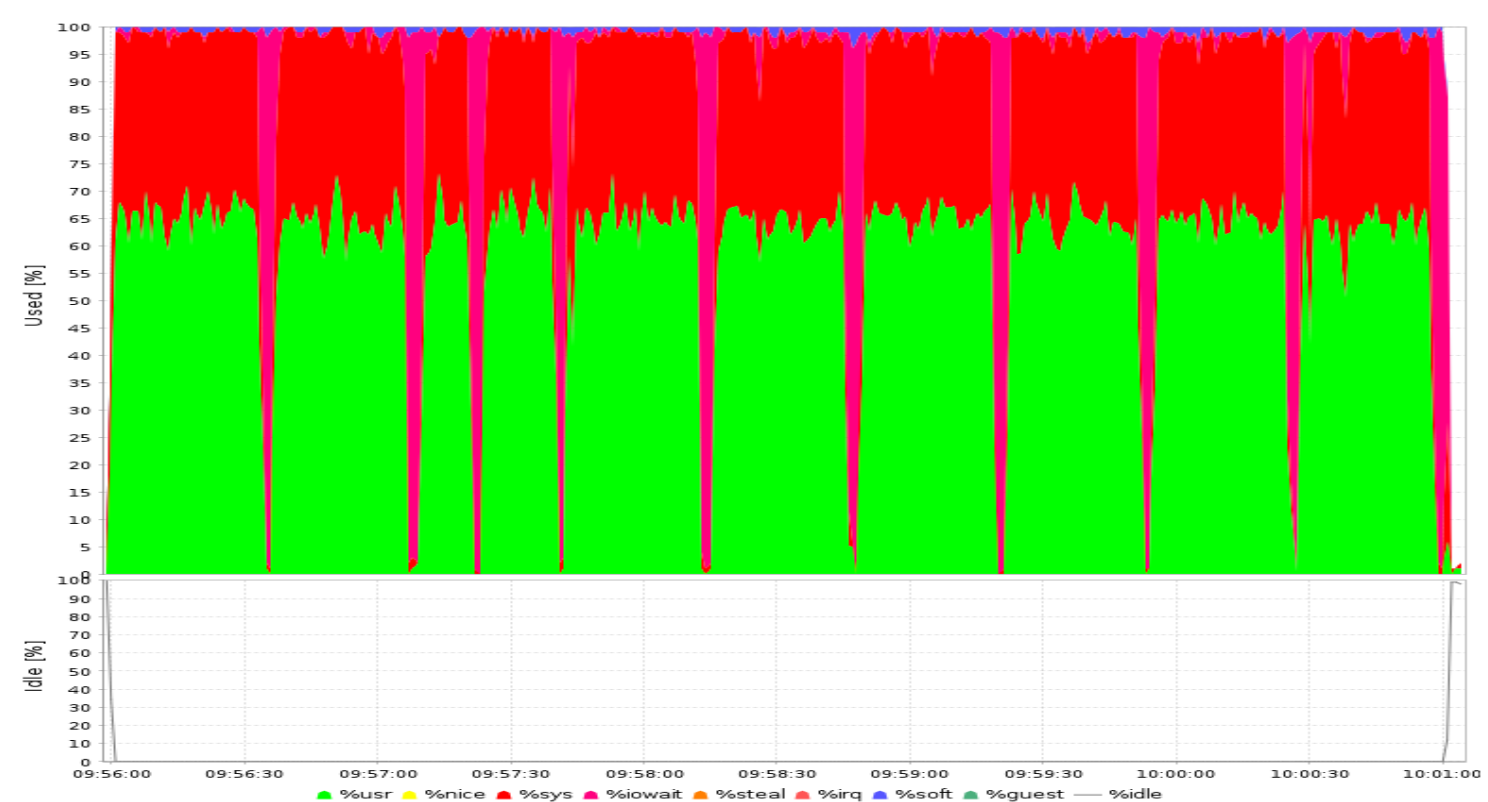

Figura 5 - Gráfico de uso da CPU pelo Vmware (Fonte:Autor)

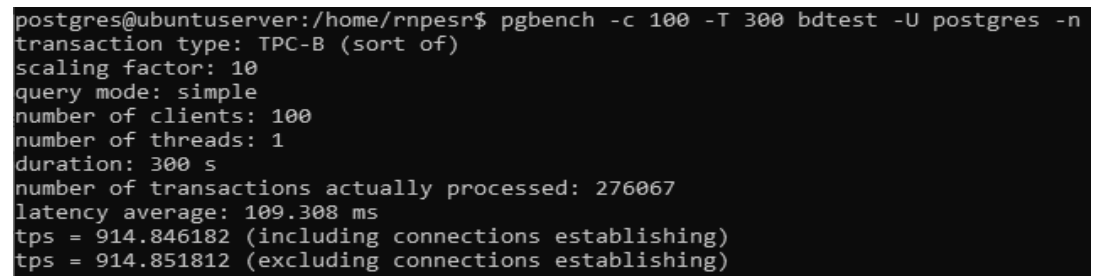

Figura 6 - Resultados de benchmark do pgbench no Vmware (Fonte:Autor)

\subsection{Xen-server}

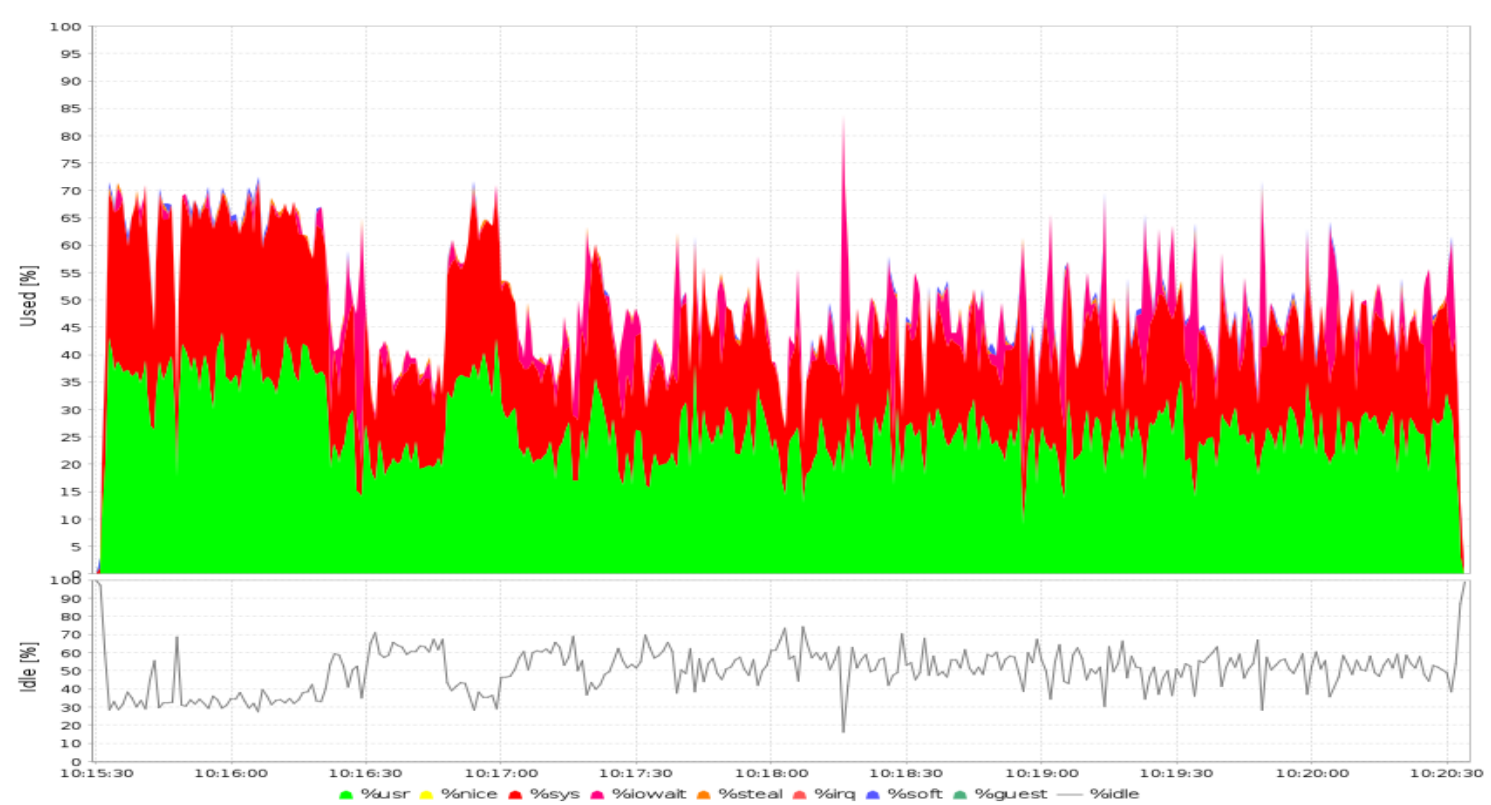

Figura 7 - Gráfico de uso da CPU pelo Xen-server (Fonte:Autor) 


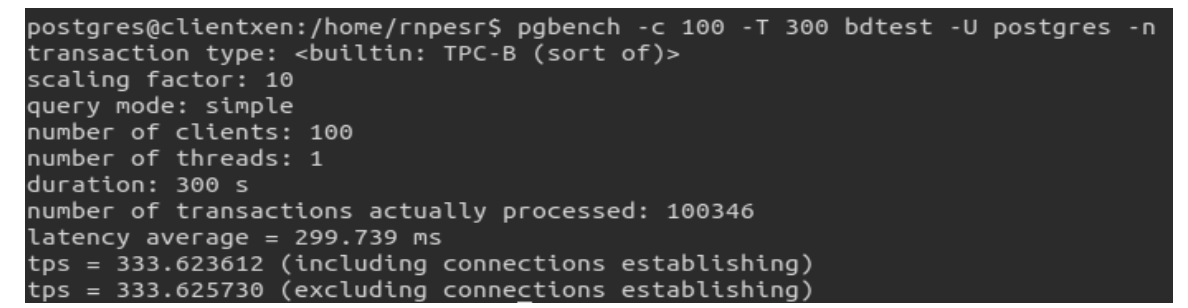

Figura 8 - Resultado de benchmark no pgbench do Xen-server (Fonte:Autor)

\section{Conclusão}

Ao realizar uma análise de todos os resultados obtidos, levando em consideração o fundamento para o qual cada virtualizador foi projetado, é possível observar uma ampla vantagem do Vmware em relação aos demais virtualizadores. demonstrando resultados superiores no pgbench, além de gerenciar melhor o uso de recursos da CPU, utilizando $100 \%$ dos recursos disponibilizados na maior parte dos testes. Os resultados obtidos pelo Xen-Server demonstram que o mesmo possui certas desvantagens em relação ao Vmware, tendo um sistema operacional, o Ubuntu Server 16.04.6 LTS neste caso, como base para a instalação do virtualizador, resultando no compartilhamento de recursos com outras aplicações. Esta mesma situação se aplica ao KVM, porém, a performance obtida e observada através dos testes, se mostra inferior ao do Xen-Server, além de demonstrar um gerenciamento de recursos que não permanece constante. A maior dificuldade foi gerenciar e instalar todos os recursos de forma que os servidores ficassem com as mesmas configurações e ajustes. Para isso todos os ajustes utilizados foram os padronizados pelas aplicações. E o hardware utilizado também possuem as mesmas configurações. Como trabalhos futuros, seria de boa valia fazer ajustes nestes virtualizadores com objetivo de uso em aplicações de banco de dados, para assim verificar se há alguma modificação nos resultados apresentados.

\section{Referências Bibliográficas}

Jung, I.H., Lee, S.H., Eom, Y.I.,Comparative Analysis of Open Source Cloud Computing Platforms. Korea Computer Congress 2012, vol. 39, nº 1, pg. 383-384.

Lee, B.Y., Park, J.H., Yoo, J.S.:Software Architecture of the Grid for implementing the Cloud Computing of the High Availability 2014. The Korea Contents Association, vol. $12, \mathrm{n}^{\circ} 2$, pg 19-29.

Mundodocker. $\mathrm{O}$ que é docker. On-line. Disponível em: $<$ http://www.mundodocker.com.br/o-que-e-docker/>. Acessado em: 04/03/2017

Seol, K., Hwang1, H., Moon1, I., Kwon1 O., Kim, B. Performance Comparison Analysis of Linux Container and Virtual Machine for Building Cloud. 2014, Advanced Science and Technology Letters. Vol.66 (Networking and Communication 2014), pg. 105-111.

Veras, M., Carissimi, A., Virtualização de Servidores, Escola superior de Redes 2015, v 2.0.0-b

Yoon, J.W., Park, C.Y., Song, U.S.:Building the Educational Practice System based on Open Source Cloud Computing. Journal of Digital Contents Society 2013, vol. 14, $\mathrm{n}^{\mathrm{o}} 4$, pg. 505-511. 